\title{
Does the Critical Shoulder Angle Correlate With Rotator Cuff Tear Progression?
}

\author{
Peter N. Chalmers MD, Dane Salazar MD, Karen Steger-May MA, \\ Aaron M. Chamberlain MD, Ken Yamaguchi MD, MBA, Jay D. Keener MD
}

Received: 8 October 2016/Accepted: 13 January 2017/Published online: 24 January 2017

(C) The Association of Bone and Joint Surgeons (B) 2017

\begin{abstract}
Background The critical shoulder angle (CSA) has been reported to be associated with rotator cuff disease and has been suggested as an etiology for cuff tears. However, it is unclear whether acromial morphologic characteristics such as CSA are a cause or effect because all studies to date have been retrospective.

Questions/purposes (1) How often can the CSA be reliably measured? (2) Is the CSA associated with rotator cuff disease? (3) Is the CSA correlated with baseline tear size or tear enlargement? (4) Does the CSA change with time? Methods In this retrospective comparison of longitudinally collected data, patients with asymptomatic rotator cuff tears underwent ultrasonography and standardized AP radiographs at enrollment and yearly thereafter during a
\end{abstract}

Funding was received from the National Institutes of Health (R01 AR051026; JDK).

One of the authors certifies that he (AMC) has or may receive payments or benefits, during the study period, an amount of USD 100,001-USD 1,000,000 from Zimmer Biomet (Warsaw, IN, USA), an amount less than USD 10,000 from Arthrex (Naples, FL, USA), and an amount less than USD 10,000 from DePuy (West Chester, PA, USA).

One of the authors certifies that he (KY) has or may receive payments or benefits, during the study period, an amount of USD 100,001-USD $1,000,000$ from Tornier (Bloomington, MN, USA) an amount of USD 10,001-100,000 from Wright Medical Technology Inc (Memphis, TN, USA) and an amount of USD 10,001-100,000 from Zimmer Biomet (Warsaw, IN, USA).

One of the authors certifies that he (JDK) has or may receive payments or benefits, during the study period, an amount of USD 100,001-1,000,000 from Zimmer Biomet (Warsaw, IN, USA), an amount less than USD 10,000 from Arthrex (Naples, FL, USA), and an amount less USD 10,000 from Genesis Shoulder Innovations (Ann Arbor, MI, USA)

All ICMJE Conflict of Interest Forms for authors and Clinical Orthopaedics and Related Research ${ }^{\circledR}$ editors and board members are on file with the publication and can be viewed on request. median of 4 years. Three hundred ninety-five patients were included, of whom 14 were excluded as they were not yet eligible for 2-year followup and $68(18 \%)$ were lost to followup, leaving 313 study patients who were evaluated with 1433 radiographs. Patients with adhesive capsulitis with normal rotator cuffs and radiographically normal scapulae were included as control subjects (119 subjects). Two observers (PNC, DS) measured the CSA in a blinded fashion. Radiographs that met Suter-Henninger criteria for CSA measurement reliability were included. For the study group, 179 of the 313 (57\%) patients with radiographs that met Suter-Henninger criteria were further analyzed; the remainder were excluded from this study. For the control group, 50 of 119 (42\%) subjects met criteria and were further analyzed. Tear enlargement was found in 94

Each author certifies that his or her institution approved the human protocol for this investigation, that all investigations were conducted in conformity with ethical principles of research, and that informed consent for participation in the study was obtained.

This study was performed at Washington University, St Louis, MO, USA.

P. N. Chalmers $(\square)$

Department of Orthopaedic Surgery, University of Utah, 590

Wakara Way, Salt Lake City, UT 84108, USA

e-mail: p.n.chalmers@gmail.com

D. Salazar, A. M. Chamberlain, K. Yamaguchi, J. D. Keener Department of Orthopaedic Surgery, Washington University, St Louis, MO, USA

K. Steger-May

Division of Biostatistics, Washington University, St Louis, MO, USA 
patients, and the CSA was compared in patients with tears and control subjects, and in tears with or without enlargement, and was correlated with tear size. In a subgroup of the study group in which 59 of 179 patients had a minimum of 3 years between initial and followup radiographs, two CSA measurements were performed to measure change.

Results In total, of the 1552 radiographs evaluated, only $326(21 \%)$ were of sufficient quality to measure the CSA. The CSA was higher among patients with cuff tears than control subjects $\left(34^{\circ} \pm 4^{\circ}\right.$ versus $32^{\circ} \pm 4^{\circ}$; mean difference, $\left.2.0^{\circ} ; 95 \% \mathrm{CI}, 0.7^{\circ}-3.2^{\circ} ; \mathrm{p}=0.003\right)$. The CSA did not correlate with baseline tear length $(\rho=0.22, p=0.090)$ or width $(\rho=0.16, p=0.229)$. The CSA was not different between tears that enlarged and those that were stable $\left(34^{\circ}\right.$ $\pm 3^{\circ}$ versus $34^{\circ} \pm 4^{\circ}$; mean difference, $0.2^{\circ}$; $95 \% \mathrm{CI}$, $-0.9^{\circ}$ to $-1.4^{\circ} ; \mathrm{p}=0.683$ ). The CSA did not change over time (CSA Time 1: mean $33^{\circ} \pm 4^{\circ}$ SD; CSA Time 2: mean $33^{\circ} \pm 4^{\circ} \mathrm{SD}$; mean difference, $-0.2^{\circ} ; 95 \% \mathrm{CI},-0.6^{\circ}$ to $0.1^{\circ} ; \mathrm{p}=0.253$ )

Conclusions Even with a longitudinal protocol, most radiographs are of insufficient quality for CSA measurement. Although patients with a history of degenerative cuff disease have higher CSA values than control subjects, the difference is small enough that it could be influenced by measurement error in practice; in any case, a difference of the magnitude we observed is likely to be clinically unimportant. The CSA is not correlated with tear size or tear progression, and does not seem to change with time. These results suggest that the CSA is unlikely to be related to rotator cuff disease.

Level of Evidence Level II, prognostic study.

\section{Introduction}

The influence of acromial anatomy on the genesis of atraumatic rotator cuff tears has long been debated $[1,3,6,8,14,21,30]$. Morphologic features of the acromium, as a potential etiology, have been shown to be unreliable [13], and there is increasing evidence that cuff disease is linked primarily to an age-related degenerative process $[19,22]$. Theories linking acromial characteristics to rotator cuff disease have fueled the debate regarding the role of extrinsic (mechanical impingement) versus intrinsic (biologic degeneration) factors in the development of rotator cuff tears [3, 19, 22, 30]. Other acromial morphologic measurements such as acromial index and the critical shoulder angle (CSA) (Fig. 1) have been proposed to be associated with the etiology of rotator cuff disease $[2,7,10,15,26]$. The CSA is a measurement that combines lateral acromial offset and glenoid inclination
$[2,5,7,15,24,26,31]$. A more-lateral acromial offset creates a more-lateral deltoid origin and, in theory, results in greater shear and lesser compressive vector of the deltoid across the glenohumeral joint [10]. In some studies, a larger acromial index, which is a measure of lateral acromial offset $[2,26]$, has been reported to be associated with rotator cuff disease [2, 3, 26, 30], whereas another study showed no such relationship [13]. Glenoid inclination also may play a role in the development of rotator cuff disease $[7,15]$. Superior glenoid inclination increases shear forces and decreases compression forces across the glenohumeral joint and may encourage proximal humeral migration and increase loads placed on the articular margin of the supraspinatus to resist the deltoid $[5,7,15,24,31]$.

The CSA has been shown to be a reliable measurement on plain radiographs $[5,26,31,32]$. Previous studies have suggested that high CSA $\left(>35^{\circ}\right)$ correlates with rotator cuff disease, whereas low CSA $\left(<30^{\circ}\right)$ correlates with osteoarthritis $[4,5,7,24,31]$. It is unclear whether lateral acromial offset or superior glenoid inclination is the dominant factor, with one recent study suggesting glenoid inclination to be dominant [7]. In addition, it is unclear whether acromial morphologic characteristics such as the CSA are a cause or effect because all studies to date have been retrospective $[2,7,10,15,26]$. Furthermore, it is unknown if the CSA is a static measurement or if this anatomic variable changes with time. An analysis of rotator cuff disease progression can provide more definitive

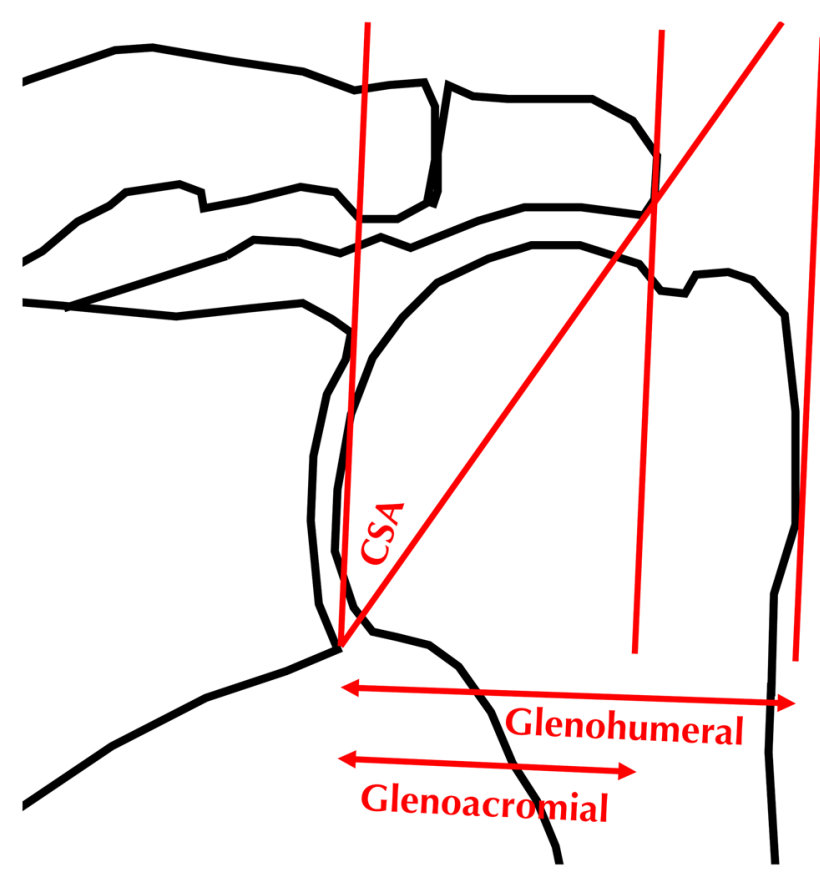

Fig. 1 This schematic shows the critical shoulder angle (CSA) and the acromial index, which is measured as the glenoacromial distance divided by the glenohumeral distance. 
evidence of the potential link of the CSA to the development and progression of degenerative rotator cuff tears.

Therefore, we asked: (1) How often can the CSA be reliably measured? (2) Is the CSA correlated with rotator cuff disease? (3) Is the CSA associated with baseline tear size or tear enlargement? (4) Does the CSA change with time?

\section{Patients and Methods}

Our university institutional review board approved this study. This study is a retrospective analysis of longitudinally collected data. The study group included is a subset of a followed group of patients with asymptomatic rotator cuff tears who presented for evaluation of shoulder pain secondary to contralateral rotator cuff disease [17, 23].

Inclusion criteria for study patients were (1) bilateral shoulder ultrasonography performed to investigate unilateral rotator cuff-based pain; (2) painful rotator cuff disease in the symptomatic shoulder; (3) absence of pain in the asymptomatic shoulder at the time of study enrollment; (4) no history of trauma to either shoulder and no traumatic episode during the study period; and (5) a minimum of either 2 years between the first study visit and final ultrasound or tear enlargement within 2 years of the first study visit (Fig. 2). This is an ongoing longitudinal protocol that has a low rate of loss of followup (18\%). Pain was specifically defined as a 0 to 10 VAS score greater than 3 for a minimum of 6 weeks, a pain level that the patient considered to be greater than regularly experienced with activities of daily living, pain that required the use of medications, or pain that prompted a visit to a physician. The absence of pain was defined as a VAS score less than 3. This threshold was selected because the senior author's (KY) clinical experience has been that patients who seek treatment generally report VAS scores greater than 5 . Exclusion criteria were (1) any past or current pain (at the time of enrollment) in the asymptomatic shoulder; (2) continuous use of narcotic or NSAIDs during the 3 months before enrollment; (3) a traumatic episode affecting the asymptomatic shoulder; (4) inflammatory arthritis; (5) radiographic evidence of osteoarthritis in the asymptomatic shoulder at the time of enrollment; (6) upper extremity weightbearing demands; and (7) an isolated subscapularis tendon tear in the asymptomatic shoulder.

Patients were enrolled from the clinical practices of three surgeons (KY, JDK, LG) during a 30-month period. The study protocol was described in depth in previous studies [17, 23]. In brief, annual standardized examinations with shoulder ultrasonography were performed. Ultrasound examinations were completed by one of two musculoskeletal radiologists (WDM, SAT) with extensive

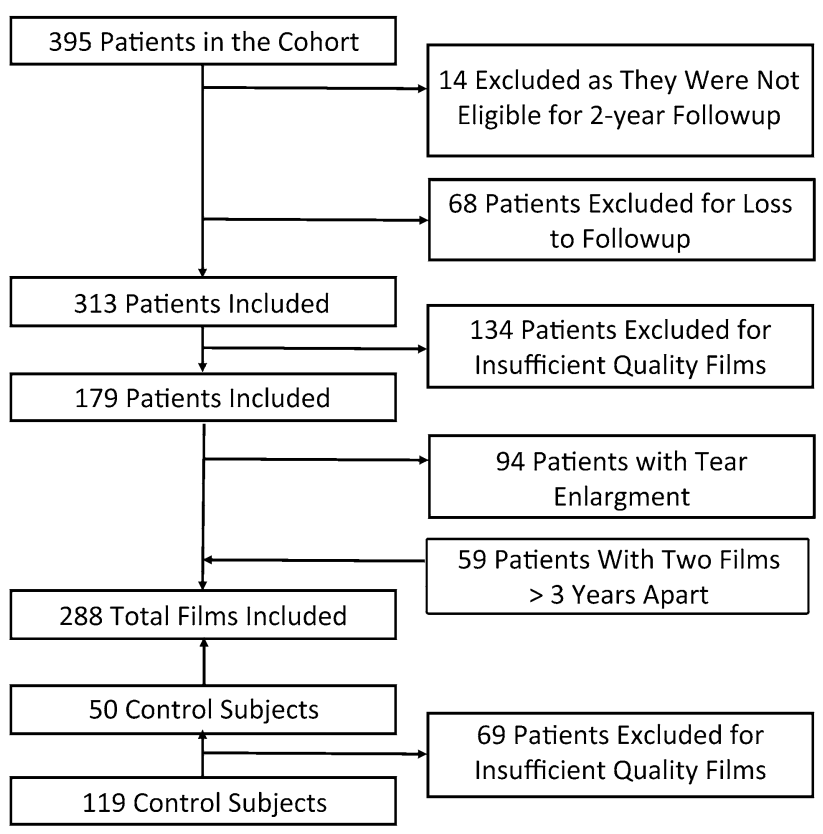

Fig. 2 This flow diagram shows the number of films and the numbers of patients included from the study cohort and the control groups after application of inclusion and exclusion criteria.

experience in the use of high-resolution ultrasonography for evaluation of pathologic conditions of the shoulder. At baseline the status of the rotator cuff in the study (asymptomatic) shoulder was classified as (1) no tear; (2) partial-thickness tear; or (3) full-thickness tear based on previously described criteria [17]. At each annual visit, the rotator cuff was reevaluated to assess tear severity (no tear, partial-thickness tear, and full-thickness tear) and tear size. For full-thickness tears, the width and length were measured [17]. Tear enlargement was defined as an increase in tear width or retraction of $5 \mathrm{~mm}$ or greater or a progressive change in tear severity (ie, partial- to full-thickness tear) similar to previous definitions of this cohort [17].

For the control group, the operative logs from the shoulder and elbow division at Washington University were reviewed and patients who underwent arthroscopic capsular release for the treatment of recalcitrant adhesive capsulitis were selected. Patients with adhesive capsulitis were selected as a control group because they would be expected to be skeletally normal. In addition, all of these patients were assessed arthroscopically to show the absence of rotator cuff disorders. Prior studies have used osteoarthritis as a control [24-26, 31], but if osteoarthritis is used as a control, it remains unclear whether differences between rotator cuff disease scapulae and osteoarthritis scapulae are the result of osteoarthritis or rotator cuff disease. Exclusion criteria for the control group included any diagnosis other than adhesive capsulitis, radiographic evidence of osteoarthritis or proximal humeral migration, 
intraoperative evidence of rotator cuff disorder, and preoperative radiographs that did not qualify as class A1 or C1 using the Suter-Henninger scale (Fig. 3) [32]. For patients who met inclusion criteria, the CSA was measured and served as a control group for the degenerative rotator cuff tear cohort.

Shoulder radiographs were obtained at baseline and annually using a standardized protocol by specifically trained technicians, as previously described [20]. These radiographs then were evaluated by two independent observers (PNC, DS) with our digital radiography software suite (PACS; General Electric, Fairfield, CT, USA) using a standardized protocol. The observers who performed the radiographic measurements were blinded to ultrasonographic and clinical findings for each subject. Only true AP radiographs were evaluated. Given that the accuracy of the CSA is influenced by subtle variances in scapular position [32], strict criteria were used to include only the highest quality radiographs. Specifically, we used the Suter-Henninger grading scale [32]. This scale grades whether the Grashey view radiograph obtained is perfectly orthogonal to the glenoid mediolaterally (type A) or not (types B, C, and D) and whether it is perfectly orthogonal to the glenoid superoinferiorly (type 1) or not (types 2 and 3) (Fig. 3). A previous study showed that excluding radiographs with types $\mathrm{A} 2 / 3, \mathrm{~B} 1 / 2 / 3, \mathrm{C} 2 / 3$, and $\mathrm{D} 1 / 2 / 3$, improves CSA accuracy such that $89 \%$ of measurements will be within less than $2^{\circ}$ of accuracy (Fig. 3) [32]. Starting with the radiograph obtained at the time of study enrollment, each yearly longitudinal radiograph was graded until an appropriate-quality radiograph, as defined by the Suter-Henninger scale, was obtained. If no adequate radiograph could be located, then the patient was excluded from the study. The CSA then was measured digitally as the angle between a line from the inferior-most subchondral glenoid to the lateral acromial edge and a line from the superior-most subchondral glenoid to the inferior-most subchondral glenoid, as previously described (Fig. 4) [5, 26, 31, 32]. Previous studies showed that the CSA can be measured reliably on plain radiographs and that it is not necessary to obtain a CT scan [5] or MRI [31] to evaluate this angle.
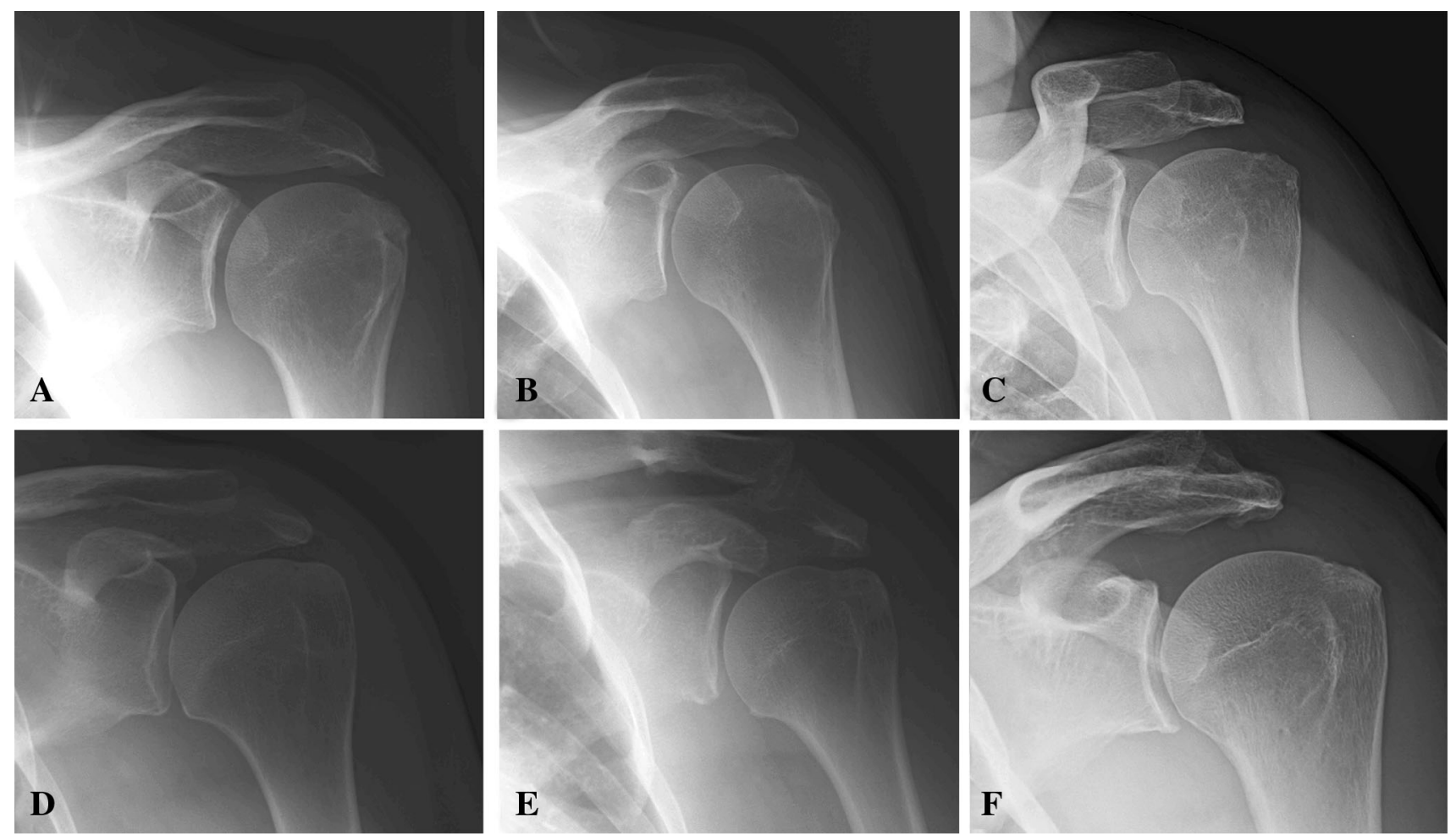

Fig. 3A-F These radiographs show the Suter-Henninger [32] classification scheme. (A) In Type A1, there is perfect overlap between the anterior and posterior glenoid rims and overlap between the coracoid and the superior glenoid pole; (B) in Type B1, there is imperfect overlap between the anterior and posterior glenoid rims in the superior $50 \%$ of the glenoid but with overlap between the coracoid and the superior glenoid pole; $(\mathbf{C})$ in Type $\mathbf{C}$, there is imperfect overlap between the anterior and posterior glenoid rims in the inferior $50 \%$ of the glenoid but with overlap between the coracoid and the superior glenoid pole; (D) in Type D1, there is imperfect overlap between the anterior and posterior glenoid rims over the entire glenoid but with overlap between the coracoid and the superior glenoid pole; $(\mathbf{E})$ in Type A2, there is perfect overlap between the anterior and posterior glenoid rims but with no overlap between the coracoid and the superior glenoid pole as the coracoid is too superior; and $(\mathbf{F})$ in Type A3, there is perfect overlap between the anterior and posterior glenoid rims but with no overlap between the coracoid and the superior glenoid pole as the coracoid is too inferior. 


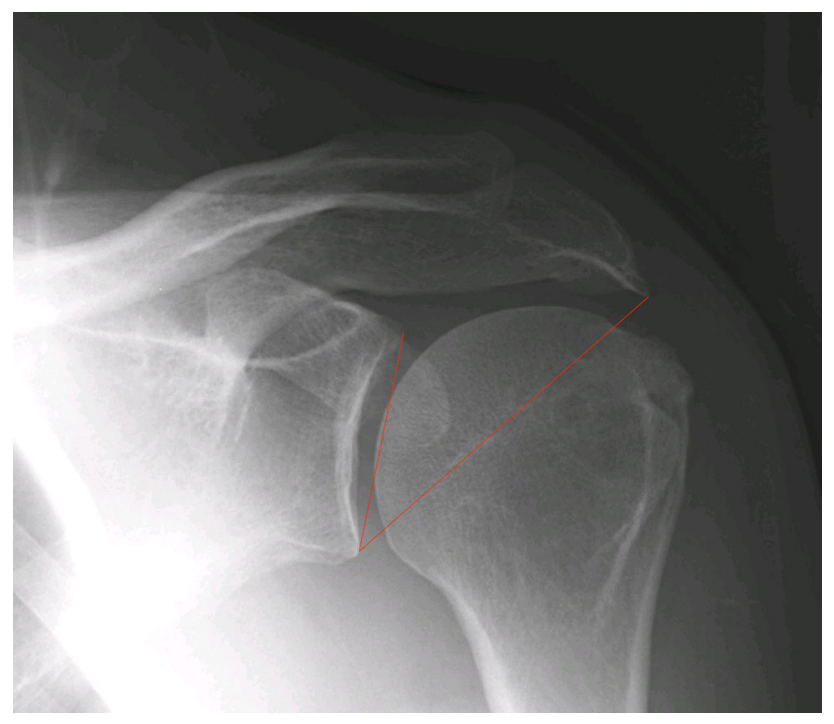

Fig. 4 This radiograph shows the method used for CSA measurement, which is measured as the angle between a line from the inferiormost subchondral glenoid to the lateral acromial edge and a line from the superior-most subchondral glenoid to the inferior-most subchondral glenoid.

In addition, patients with multiple radiographs that met the criteria were included as a subset from our cohort to determine potential changes in the CSA with time. The most recent annual radiograph of sufficient quality served as a comparison to the radiograph obtained at the time of enrollment (or the closest temporal radiograph of sufficient quality). The minimum time between these radiographs had to be 3 years or greater to qualify as adequate comparison films.

For the study group, a total 313 subjects had sufficient 2year followup from the time of enrollment (Fig. 2). The median duration of followup was 4.5 years (interquartile range, 0-9.6). From this cohort, one subject was excluded because a defibrillator obscured the glenohumeral joint on all available radiographs. From the remaining subjects, 1433 films were evaluated. For the control group, 119 patients with adhesive capsulitis who had undergone capsular release met all inclusion criteria and had radiographs obtained at our institution. The CSA was evaluated by two reviewers (PNC, DS) on 288 total radiographs $(\mathrm{n}=238$ study group; $\mathrm{n}=50$ control subjects). An analysis was conducted comparing patients who were excluded with those who were included who showed no difference in tear type $(p=0.558)$, age $(p=0.503)$, tear length $(p=0.603)$, tear width $(\mathrm{p}=0.208)$, tear length among full-thickness tears $(p=0.806)$, tear width among full-thickness tears $(p=$ $1.000)$, but who did show a difference in gender $(53 \%$ males in the excluded patients vs $63 \%$ males in the included patients, $\mathrm{p}=0.036$ ).
Statistical Analysis

Interobserver reliability was measured with intraclass correlation coefficients (ICCs) and associated 95\% CIs. The ICC for these two reviewers was 0.88 (95\% CI, 0.86-0.91) with an ICC of 0.93 (95\% CI, 0.88-0.96) for the control group and 0.87 (95\% CI, 0.84-0.90) for the study group. Thereafter, the mean of the two CSA measurements was used for analyses. Of 179 patients, 59 had a minimum of 3 years between films, and changes in CSA with time were evaluated in these 59 patients with the Wilcoxon signedrank test. Patients were divided into four groups: partialthickness tears, full-thickness tears, study control subjects (ie, patients with a contralateral but not ipsilateral rotator cuff tear), and control subjects (ie, patients with adhesive capsulitis). These groups were compared using ANOVA. Pairwise comparisons among each of the four groups were performed using least squares means that are Tukey-adjusted for multiple comparisons. A statistical contrast in the ANOVA was used to compare the combined partial- and full-thickness tears with each control group. Patients in the rotator cuff tear group were divided into those with (94/ 179) and without (84/179) tear enlargement during the study period and CSA was compared using a Welch's ANOVA test. Among patients with full-thickness tears (59/ 179), baseline tear length and width (in $\mathrm{mm}$ ), and change (calculated by subtracting baseline from the final study measurement) in tear length and width were correlated with the CSA using Spearman's correlation coefficients $(\rho)$.

\section{Results}

\section{How Often Can the CSA be Reliably Measured?}

In total, of the 1552 radiographs evaluated only $326(21 \%)$ were of sufficient quality to measure the CSA. In the study group, using the Suter-Henninger grading scale, 20\% (280/ 1433) of radiographs were Class A, $3 \%$ (41/1433) were Class B, 1\% (19/1433) were Class C, 76\% (1093/1433) were Class D, 96\% (1379/1433) were Type 1, 1\% (18/ $1433)$ were Type 2 , and $3 \%(36 / 1433)$ were Type 3 . In total, only $19 \%(276 / 1433)$ of films were A1 or C1 radiographs. Of the 312 potential subjects, 179 (57\%) had an A1 or $\mathrm{C} 1$ radiograph at any point during the study. In the control group, 50 shoulders $(42 \%, 50 / 119)$ had an A1 or C1 quality radiograph.

\section{Is the CSA Associated With Rotator Cuff Disease?}

The mean CSA value for the study group $\left(34^{\circ} \pm 4^{\circ}\right)$ was greater than that of the control group $\left(32^{\circ} \pm 4^{\circ}\right.$; mean 
Table 1. Association between CSA and tear severity at enrollment

\begin{tabular}{llll}
\hline Group & CSA & $\begin{array}{l}\text { Mean difference compared } \\
\text { with control subjects (95\% CI) }\end{array}$ & $\begin{array}{l}\text { p Value compared with } \\
\text { control subjects }\end{array}$ \\
\hline Control $(\mathrm{n}=50)$ & $31.7^{\circ} \pm 4.3^{\circ}$ & Reference & Reference \\
Study groups $(\mathrm{n}=178)$ & $33.7^{\circ} \pm 3.8^{\circ}$ & $2.0^{\circ}\left(0.7^{\circ}-3.2^{\circ}\right)$ & 0.003 \\
No tear $(\mathrm{n}=37)$ & $33.8^{\circ} \pm 3.6^{\circ}$ & $2.1^{\circ}\left(-0.1^{\circ}\right.$ to $\left.4.3^{\circ}\right)$ & 0.062 \\
Tear $(\mathrm{n}=141)$ & $33.6^{\circ} \pm 3.9^{\circ}$ & $1.9^{\circ}\left(0.4^{\circ}-3.4^{\circ}\right)$ & 0.005 \\
$\quad$ Partial tear $(\mathrm{n}=51)$ & $33.2^{\circ} \pm 3.1^{\circ}$ & $1.5^{\circ}\left(-0.5^{\circ}\right.$ to $\left.3.5^{\circ}\right)$ & 0.228 \\
Full tear $(\mathrm{n}=90)$ & $33.9^{\circ} \pm 4.2^{\circ}$ & $2.2^{\circ}\left(0.4^{\circ}-3.9^{\circ}\right)$ & 0.010 \\
\hline
\end{tabular}

Data presented as mean $\pm \mathrm{SD}$.

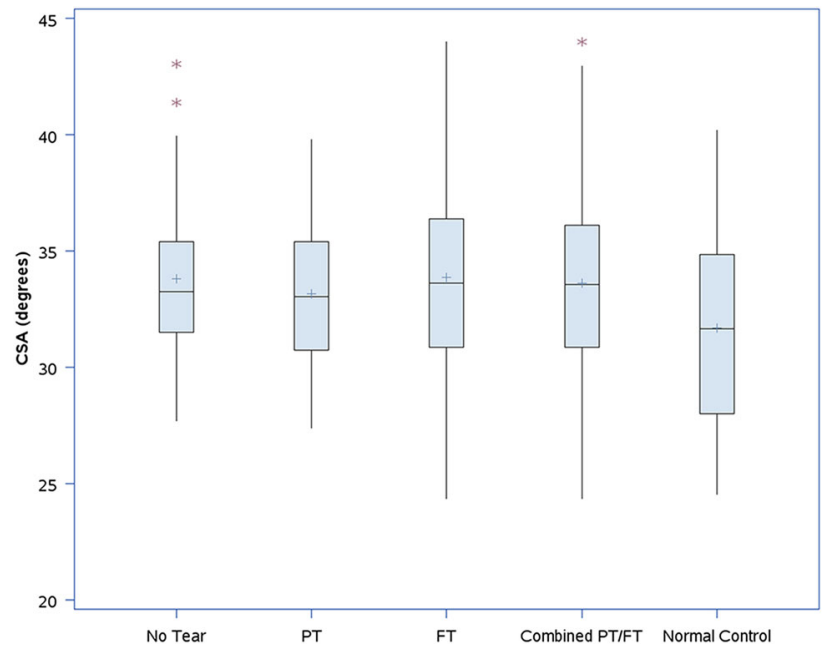

Fig. 5 This box plot shows CSA measurements for patients with no tear, study patients with partial-thickness (PT) tears, full-thickness (FT) tears, and combined partial- and full-thickness tears, and control subjects. The boxes represent the interquartile range, with the central line representing the median and the plus sign representing the mean. The whiskers represent the furthest value within 1.5 times of the interquartile range. The values between 1.5 and three times the IQR are denoted with asterisks. There are no values that are more than three times the interquartile range.

difference, $\left.2.0^{\circ} ; 95 \% \mathrm{CI}, 0.7^{\circ}-3.2^{\circ} ; \mathrm{p}=0.003\right)$. Subgroup analysis showed the CSA was higher for the patients with combined partial- and full-thickness rotator cuff tears than the control subjects $\left(33.6^{\circ} \pm 3.9^{\circ}\right.$; mean difference $1.9^{\circ}$; $95 \% \mathrm{CI},-0.4^{\circ}$ to $3.4^{\circ} ; \mathrm{p}=0.005$ ) (Table 1 ; Fig. 5). This difference was driven by the difference between fullthickness tears and control shoulders $(p=0.010)$ because there was no difference between control subjects and patients with partial-thickness tears $(\mathrm{p}=0.228)$ (Table 1). Subgroup analysis in the study group showed no difference in the CSA for shoulders with no tears compared with shoulders with partial- or full-thickness tears or the combined group of partial- and full-thickness tears $(\mathrm{p}=0.87$, 0.99 , and 0.68 , respectively).
Is the CSA Associated With Baseline Tear Size or Tear Enlargement?

In patients with full-thickness tears, the CSA did not correlate with baseline tear length $(\rho=0.22, p=0.090)$ or width $(\rho=0.16, p=0.229)$. There was no difference in the CSA between the groups with partial- and full-thickness rotator cuff tears $(p=0.113)$ that later enlarged. For shoulders with tear enlargement, smaller initial CSA values slightly correlated with an increase in tear length with time ( $\rho=-0.28, p=0.043)$, whereas larger initial CSA values slightly correlated with an increase in tear width $(\rho=0.29$, $p=0.028)$. In the study groups, 94 shoulders showed tear enlargement during surveillance (median time to enlargement from enrollment, 3.3 years; interquartile range, 0-7.6 years). When comparing the risk of tear enlargement for study shoulders compared with the shoulders that were excluded owing to inadequate radiographs, there was no difference between groups (53\% versus 59\%; $\mathrm{p}=0.314$ ). When examining tear progression, when patients were divided into those with tear enlargement $(n=94)$ compared with those with stable tears $(n=84)$, there were no differences in the CSA between groups $\left(34^{\circ} \pm 3^{\circ}\right.$ versus $34^{\circ}$ $\pm 4^{\circ}$; mean difference, $0.2^{\circ} ; 95 \% \mathrm{CI},-0.9^{\circ}$ to $1.4^{\circ} ; \mathrm{p}=$ 0.683 ). Finally, to confirm that rotator cuff progression was not associated with especially large CSAs, we performed a subgroup analysis comparing patients with a CSA larger than $35^{\circ}$ with those with a CSA smaller than $35^{\circ}$. Tear enlargement was not significantly different for patients with a large CSA (CSA $\left.>35^{\circ} ; n=59\right)$ and patients without a large CSA $\left(\mathrm{CSA} \leq 35^{\circ} ; \mathrm{n}=119\right)(54 \%$ versus $52 \%$; $\mathrm{p}=$ 0.788 by chi-square test). A median split of CSA also was performed to create two approximately equal-size groups of patients. Tear enlargement is not significantly different for patients with a large CSA $\left(\mathrm{CSA}>33.4^{\circ} ; \mathrm{n}=90\right)$ and patients without a large CSA $\left(\mathrm{CSA} \leq 33.4^{\circ} ; \mathrm{n}=88\right)(53 \%$ versus $52 \%$; $=0.887$ by chi-square test). A post hoc power analysis was performed for this specific analysis. With the observed mean CSA and observed variability in each group, the study sample sizes of 84 patients without 
and 94 patients with tear enlargement would have $80 \%$ statistical power to detect a mean CSA between-group difference of $1.6^{\circ}$ with a two-tailed test at $0.05 \alpha$ if that difference was achieved.

\section{Does the CSA Change With Time?}

In the 33\% (59 of 179) of patients with adequate radiographs separated by at least 3 years, the CSA did not change (CSA Time 1: mean, $33^{\circ} \pm 4^{\circ} \mathrm{SD}$; CSA Time 2: mean, $33^{\circ} \pm 4^{\circ} \mathrm{SD}$; mean difference, $-0.2^{\circ} ; 95 \% \mathrm{CI}$, $-0.6^{\circ}$ to $0.1^{\circ} ; \mathrm{p}=0.253$ ). A post hoc power analysis was performed for this specific analysis. With the observed mean CSA and observed variability, the study would have $80 \%$ power to detect an absolute change of at least $0.491^{\circ}$ if that change was observed.

\section{Discussion}

Although the prevailing theories regarding the etiology of degenerative rotator cuff tears primarily have suggested an age-related, intrinsic tendon process [19, 22], the potential importance of the skeletal anatomy has been studied for decades and remains a controversial subject $[3,8,14,21,30]$. We sought to examine the potential role of the CSA as a risk factor for the development and progression of atraumatic degenerative rotator cuff tears, which may provide valuable information regarding the natural progression of rotator cuff disease and help to identify higher-risk patients. The findings of this study suggest that morphologic features of the scapula, as defined by the CSA, are associated with the presence of degenerative rotator cuff tears; however, the CSA has no apparent bearing on the risk of tear progression at moderateterm followup. Finally, we found no change in the CSA at a mean of 6 years, suggesting this is a stable measurement.

Our study has several limitations. The CSA may be affected by heterogeneities in patient positioning, gantry and plate positioning, and scapular positioning, all of which may limit reliability [24, 32]. To mitigate this effect, we used a standardized protocol and a previously validated grading scale to allow exclusion of suboptimal radiographs [32]. In our data set, which was drawn from a standardized radiographic set with stringent criteria, this protocol still required the exclusion of $43 \%$ of potential patients. Our analysis showed no difference in the percentage of patients with tear enlargement between those with excluded radiographs and nonexcluded radiographs, suggesting that these exclusions did not bias our results. Past studies have not used these criteria and thus their findings are in question $[2,7,10,15,26]$. Despite our strict radiographic exclusion criteria, to our knowledge, this is the largest series to date examining the CSA in a cuff disease cohort. Another limitation relates to the temporal relationship of ultrasound measurements compared with radiographic measures. Owing to the exclusion of some radiographs, the exact timing of these two studies did not always correlate during followup. We believe this issue had little to no influence on our findings given the lack of change in the CSA observed with time. Another limitation was inclusion of patients with adhesive capsulitis as a control group instead of patients without any shoulder disorders. However, because these patients were confirmed arthroscopically to have an intact rotator cuff, they serve as an adequate control group to address our research question. An additional limitation is the relatively short followup.

\section{How Often Can the CSA be Reliably Measured?}

The CSA could not be reliably measured on most radiographs. We used strict criteria so as to include only the highest-quality radiographs for this study. Previous research has shown that subtle variance in scapular position can influence the variability and accuracy of CSA measurements [32]. Suter et al. [32] described a more-profound effect of altered scapular position on the accuracy of CSA measurements compared with that in a previous study [24]. The sensitivity of CSA to scapular position, as described by Suter et al. [32], was similar in magnitude to the mean difference in the CSA $\left(2^{\circ}\right)$ between our study and control groups. Despite the use of a longstanding radiographic protocol with specifically trained radiology technicians, $43 \%$ of potential patients were excluded owing to inadequate films and only $19 \%$ of all films met criteria for inclusion. Future research using CSA measurements should carefully control for radiograph quality to maximize accuracy. Research that did not apply these criteria may not have reliably measured the CSA $[2,7,10,15,26]$.

\section{Is the CSA Associated With Rotator Duff Disease?}

Although our findings suggest a difference in the CSA between patients with rotator cuff tears and control subjects, numerically the differences between these groups in our cohort are smaller than in prior studies $[2,7,10,15,26]$. We believe the association between the CSA and degenerative cuff disease should be interpreted with caution. A $2^{\circ}$ difference in CSA between groups is likely small enough to be clinically unimportant, which raises concerns regarding the importance of morphologic features of the scapula as risk factors for degenerative cuff disease. In addition, a $2^{\circ}$ difference is likely within measurement error, suggesting that the CSA may not be useful 
as a prognostic factor in predicting degenerative cuff disease. The mean CSA of our study group was $34^{\circ}$. Moor et al. $[24,26]$ suggested CSA values greater than $35^{\circ}$ are associated with rotator cuff disease (mean, $38^{\circ}$ ), whereas CSA values less than $30^{\circ}$ are associated with glenohumeral osteoarthritis. The mean CSA of our patients with cuff disease actually corresponds to the values of their control shoulders. Theoretically, there should be few differences in measuring the CSA between studies so long as careful control of radiographic quality is used. To our knowledge, our study is the first to use the Suter-Henninger criteria, which may help explain the differences in the absolute values and numerically smaller differences in the CSA between shoulders with rotator cuff tears and control shoulders in our study. Several cross-sectional studies have shown that patients with rotator cuff tears have a larger CSA than control subjects [24], shoulders with nonrotator cuff and nonosteoarthritic disorders [4, 26, 31], and shoulders with glenohumeral osteoarthritis [4, 24, 31]. Patients with glenohumeral osteoarthritis may not be the best comparison group because glenoid declination may be part of the osteoarthritic deformity progression [11]. Moor et al. [25] suggested the CSA is a better predictor of rotator cuff disorders than age or a history of trauma. Furthermore, the CSA also may correlate with tear size [4] and might be larger in patients with degenerative cuff tears than in patients with traumatic tears [2]. Given the apparent association of cuff disease and the CSA, a fundamental question relates to the relative importance of glenoid inclination compared with lateral acromial extension. Some studies have suggested superior glenoid inclination to be associated with rotator cuff tears [7, 15]; however, there are conflicting conclusions regarding the acromial index (a surrogate measure of lateral acromial extension). Moor et al. [26] proposed a link between acromial index and degenerative rotator cuff tears; however, a previous analysis of patients from this cohort showed similar CSA values between patients with degenerative cuff tear and control subjects [13]. Further research is needed to investigate the more-important scapular structural features that may predispose patients to rotator cuff disease.

\section{Is the CSA Associated With Baseline Tear Size or Tear} Enlargement?

Perhaps the most clinically relevant finding from this study is the lack of correlation of the CSA with tear severity or the risk of tear enlargement at mid-term followup. The CSA did not correlate with baseline dimensions, did not differ between patients with partial- and full-thickness tears at the time of enrollment, and did not differ between patients with tear enlargement and those with stable tears.
A post hoc power analysis showed that our study had $80 \%$ power to detect a difference of $1.6^{\circ}$ between patients with tear enlargement and those without tear enlargement, whereas only a $0.3^{\circ}$ difference between these groups was found. We suggest that a difference less than $1.6^{\circ}$ is not clinically meaningful and is smaller than the reported differences between control subjects and patients with rotator cuff tears in prior studies [24, 26]. Therefore, our study was adequately powered to detect a clinically meaningful difference should one have existed. Although the CSA did correlate with absolute changes in tear dimensions for shoulders with tear enlargement, the correlation between changes in tear length and CSA was negative and the correlation between tear changes in width and CSA was positive. We can offer no biologic explanation for these findings. Our study findings support the concept that although there is an association of CSA with degenerative cuff disease, the CSA is not associated with tear size progression at mid-term followup.

\section{Does the CSA Change With Time?}

In our cohort, the CSA did not change with time at midterm followup suggesting this to be a developmental skeletal feature and not an acquired degenerative deformity. A post hoc power analysis showed that our study had $80 \%$ power to detect a difference of $0.5^{\circ}$ between patients with tear enlargement and those without tear enlargement, whereas only a $0.2^{\circ}$ difference between these groups was found. A difference less than $0.5^{\circ}$ is not clinically meaningful and is smaller than the reported differences between control subjects and patients with rotator cuff tears in prior studies [24, 26]. Therefore, our study was adequately powered to detect a clinically meaningful difference should one have existed. To our knowledge, no previous studies have examined for CSA progression with time; however, some studies have shown that rotator cuff tears are progressive [18, 23, 27]. Rotator cuff tears also can lead to a characteristic series of degenerative changes including proximal humeral migration, superior wear, and inferior osteophyte formation, which are collectively termed rotator cuff tear arthropathy $[12,28]$. Studies with long-term followup have shown that these changes are progressive $[9,16,29,33]$. The combination of superior glenoid wear and inferior osteophyte formation can increase superior glenoid inclination. Further studies with longer-term followup will be necessary to understand the effect of these changes on CSA. In our data set, the CSA did not change and thus may have a causal association rather than be representative of an effect of rotator cuff disease.

Even with a longitudinal protocol, most radiographs are of insufficient quality for CSA measurement. Although 
patients with a history of degenerative cuff disease do have higher CSA values than control subjects, the difference is small enough that it could be influenced by measurement error in practice; in any case, a difference of the magnitude we observed is likely to be clinically unimportant. The CSA is not correlated with tear size or tear progression, and does not seem to change with time. These results suggest that the CSA is unlikely to be related to rotator cuff disease. Future studies will be necessary to understand whether other aspects of morphologic features of the scapula, such as glenoid inclination, may be more closely related to the development of rotator cuff disease.

Acknowledgments We thank Leesa Galatz MD (Department of Orthopaedic Surgery, Mount Sinai School of Medicine, New York, $\mathrm{NY}$ ), for contributing patients to the study. We also thank William D. Middleton MD and Sharlene A. Teefey MD (Mallinckrodt Institute of Radiology, Washington University School of Medicine) for assistance with musculoskeletal ultrasonography.

\section{References}

1. Abrams GD, Gupta AK, Hussey KE, Tetteh ES, Karas V, Bach BR Jr, Cole BJ, Romeo AA, Verma NN. Arthroscopic repair of full-thickness rotator cuff tears with and without acromioplasty: randomized prospective trial with 2-year follow-up. Am J Sports Med. 2014;42:1296-1303.

2. Balke M, Liem D, Greshake O, Hoeher J, Bouillon B, Banerjee M. Differences in acromial morphology of shoulders in patients with degenerative and traumatic supraspinatus tendon tears. Knee Surg Sports Traumatol Arthrosc. 2016;24:2200-2205.

3. Bigliani LU, Levine WN. Subacromial impingement syndrome. $J$ Bone Joint Surg Am. 1997;79:1854-1868.

4. Blonna D, Giani A, Bellato E, Mattei L, Caló M, Rossi R, Castoldi F. Predominance of the critical shoulder angle in the pathogenesis of degenerative diseases of the shoulder. J Shoulder Elbow Surg. 2016;25:1328-1336.

5. Bouaicha S, Ehrmann C, Slankamenac K, Regan WD, Moor BK. Comparison of the critical shoulder angle in radiographs and computed tomography. Skeletal Radiol. 2014;43:1053-1056.

6. Chahal J, Mall N, MacDonald PB, Van Thiel G, Cole BJ, Romeo AA, Verma NN. The role of subacromial decompression in patients undergoing arthroscopic repair of full-thickness tears of the rotator cuff: a systematic review and meta-analysis. Arthroscopy. 2012;28:720-727.

7. Daggett M, Werner B, Collin P, Gauci MO, Chaoui J, Walch G. Correlation between glenoid inclination and critical shoulder angle: a radiographic and computed tomography study. J Shoulder Elbow Surg. 2015;24:1948-1953.

8. Farfaras S, Sernert N, Hallström E, Kartus J. Comparison of open acromioplasty, arthroscopic acromioplasty and physiotherapy in patients with subacromial impingement syndrome: a prospective randomised study. Knee Surg Sports Traumatol Arthrosc. 2016:24:2181-2191.

9. Gerber C, Rahm SA, Catanzaro S, Farshad M, Moor BK. Latissimus dorsi tendon transfer for treatment of irreparable posterosuperior rotator cuff tears: long-term results at a minimum follow-up of ten years. J Bone Joint Surg Am. 2013;95:19201926.

10. Gerber C, Snedeker JG, Baumgartner D, Viehöfer AF. Supraspinatus tendon load during abduction is dependent on the size of the critical shoulder angle: a biomechanical analysis. $J$ Orthop Res. 2014;32:952-957.

11. Habermeyer P, Magosch P, Luz V, Lichtenberg S. Three-dimensional glenoid deformity in patients with osteoarthritis: a radiographic analysis. J Bone Joint Surg Am. 2006;88:13011307.

12. Hamada K, Fukuda H, Mikasa M, Kobayashi Y. Roentgenographic findings in massive rotator cuff tears: a long-term observation. Clin Orthop Relat Res. 1990;254:92-96.

13. Hamid N, Omid R, Yamaguchi K, Steger-May K, Stobbs G, Keener JD. Relationship of radiographic acromial characteristics and rotator cuff disease: a prospective investigation of clinical, radiographic, and sonographic findings. J Shoulder Elbow Surg. 2012;21:1289-1298.

14. Henkus HE, de Witte PB, Nelissen RG, Brand R, van Arkel ER. Bursectomy compared with acromioplasty in the management of subacromial impingement syndrome: a prospective randomised study. J Bone Joint Surg Br. 2009;91:504-510.

15. Hughes RE, Bryant CR, Hall JM, Wening J, Huston LJ, Kuhn JE, Carpenter JE, Blasier RB. Glenoid inclination is associated with full-thickness rotator cuff tears. Clin Orthop Relat Res. 2003;407:86-91.

16. Jost B, Zumstein M, Pfirmann CW, Gerber C. Long-term outcome after structural failure of rotator cuff repairs. J Bone Joint Surg Am. 2006;88:472-479.

17. Keener JD, Galatz LM, Teefey SA, Middleton WD, Steger-May K, Stobbs-Cucchi G, Patton R, Yamaguchi K. A prospective evaluation of survivorship of asymptomatic degenerative rotator cuff tears. J Bone Joint Surg Am. 2015;97:89-98.

18. Keener JD, Hsu JE, Steger-May K, Teefey SA, Chamberlain AM, Yamaguchi K. Patterns of tear progression for asymptomatic degenerative rotator cuff tears. $J$ Shoulder Elbow Surg. 2015;24:1845-1851.

19. Keener JD, Steger-May K, Stobbs G, Yamaguchi K. Asymptomatic rotator cuff tears: patient demographics and baseline shoulder function. J Shoulder Elbow Surg. 2010;19:1191-1198.

20. Keener JD, Wei AS, Kim HM, Steger-May K, Yamaguchi K. Proximal humeral migration in shoulders with symptomatic and asymptomatic rotator cuff tears. $J$ Bone Joint Surg Am. 2009;91:1405-1413.

21. Ketola S, Lehtinen J, Arnala I, Nissinen M, Westenius H, Sintonen $\mathrm{H}$, Aronen P, Konttinen YT, Malmivaara A, Rousi T. Does arthroscopic acromioplasty provide any additional value in the treatment of shoulder impingement syndrome?: a two-year randomised controlled trial. J Bone Joint Surg Br. 2009;91:1326-1334.

22. Kim HM, Dahiya N, Teefey SA, Middleton WD, Stobbs G, Steger-May K, Yamaguchi K, Keener JD. Location and initiation of degenerative rotator cuff tears: an analysis of three hundred and sixty shoulders. J Bone Joint Surg Am. 2010;92:1088-1096.

23. Mall NA, Kim HM, Keener JD, Steger-May K, Teefey SA, Middleton WD, Stobbs G, Yamaguchi K. Symptomatic progression of asymptomatic rotator cuff tears: a prospective study of clinical and sonographic variables. J Bone Joint Surg Am. 2010;92:2623-2633.

24. Moor BK, Bouaicha S, Rothenfluh DA, Sukthankar A, Gerber C. Is there an association between the individual anatomy of the scapula and the development of rotator cuff tears or osteoarthritis of the glenohumeral joint?: a radiological study of the critical shoulder angle. Bone Joint J. 2013;95:935-941.

25. Moor BK, Röthlisberger M, Müller DA, Zumstein MA, Bouaicha S, Ehlinger M, Gerber C. Age, trauma and the critical shoulder angle accurately predict supraspinatus tendon tears. Orthop Traumatol Surg Res. 2014;100:489-494.

26. Moor BK, Wieser K, Slankamenac K, Gerber C, Bouaicha S. Relationship of individual scapular anatomy and degenerative rotator cuff tears. J Shoulder Elbow Surg. 2014;23:536-541. 
27. Moosmayer S, Tariq R, Stiris M, Smith HJ. The natural history of asymptomatic rotator cuff tears: a three-year follow-up of fifty cases. J Bone Joint Surg Am. 2013;95:1249-1255.

28. Neer CS 2nd, Craig EV, Fukuda H. Cuff-tear arthropathy. J Bone Joint Surg Am. 1983;65:1232-1244.

29. Paxton ES, Teefey SA, Dahiya N, Keener JD, Yamaguchi K, Galatz LM. Clinical and radiographic outcomes of failed repairs of large or massive rotator cuff tears: minimum ten-year followup. J Bone Joint Surg Am. 2013;95:627-632.

30. Rockwood CA, Lyons FR. Shoulder impingement syndrome: diagnosis, radiographic evaluation, and treatment with a modified Neer acromioplasty. J Bone Joint Surg Am. 1993;75:409-424.
31. Spiegl UJ, Horan MP, Smith SW, Ho CP, Millett PJ. The critical shoulder angle is associated with rotator cuff tears and shoulder osteoarthritis and is better assessed with radiographs over MRI. Knee Surg Sports Traumatol Arthrosc. 2016;24:2244-2251.

32. Suter T, Gerber Popp A, Zhang Y, Zhang C, Tashjian RZ, Henninger HB. The influence of radiographic viewing perspective and demographics on the critical shoulder angle. $J$ Shoulder Elbow Surg. 2015;24:e149-158.

33. Zumstein MA, Jost B, Hempel J, Hodler J, Gerber C. The clinical and structural long-term results of open repair of massive tears of the rotator cuff. J Bone Joint Surg Am. 2008;90:2423-2431. 